Recepción: 16 / 04/ 2018

Aceptación: 04 / 06 / 2018

Publicación: 15 / 09 / 2018

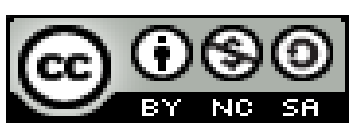

Ciencias de la Educación

Artículo de investigación

\title{
El clima organizacional y su influencia en el desempeño académico de los docentes de la Universidad Laica Eloy Alfaro de Manabí
}

\author{
The organizational climate and its influence on the academic \\ performance of teachers at the Laica Eloy Alfaro de Manabi University
O clima organizacional e sua influência no desempenho acadêmico de professores da Universidade Laica Eloy Alfaro de Manabí

\author{
Edwin A. Delgado-Veliz ${ }^{\mathrm{I}}$ \\ edaldv91@hotmail.com \\ Marcos I. Granda-García ${ }^{I I}$ \\ marco.granda@uleam.e \\ du.ec
}

Temistocles D. Loor- Chávez ${ }^{\text {III }}$

temístocles.loor@uleam.edu.ec

\author{
Wagner O. Villafuerte-Muñoz ${ }^{\mathrm{IV}}$ \\ wagnermatr38@gmail.com
}

Walter H. Villamarín-Villota ${ }^{\mathrm{V}}$

wvillamarin@gmail.com

Rafer Henry Cruz Mera ${ }^{\text {VI }}$

rafer.cruz@uleam.edu.ec

\section{Correspondencia: $\underline{\text { edaldv91@hotmail.com }}$}

I. Ingeniero en Marketing; Polo de Capacitación, Investigación y Publicación, POCAIP, Manta, Ecuador.

II. Ingeniero en Ciencias Empresariales con Especialización en Gestión Empresarial, Técnico Superior Especialización Gestión Empresarial, Docente de la Docente de la Universidad Laica Eloy Alfaro de Manabí, Manta, Ecuador.

III. Ingeniero Comercial, Magister en Dirección Universitaria, Diploma Superior en Evaluación Institucional, Diploma Superior en Enseñanza por Competencias, Especialista en Pedagogía Universitaria, Doctor en Ciencias Administrativas, Docente de la Universidad Laica "Eloy Alfaro" de Manabí, Manta , Ecuador.

IV. Diploma Superior en Gestión Directiva, Magíster en Enseñanza del Idioma Ingles, Licenciado en Ciencias de la Educación Mención en Inglés, Docente de la Universidad Laica Eloy Alfaro de Manabí, Manta, Ecuador.

v. Economista Agrícola; Magister en Finanzas y Comercio Internacional; Docente de la Universidad Laica Eloy Alfaro de Manabí, Manta, Ecuador.

VI. Ingeniero Comercial; Magister en Gerencia Educativa, Docente de la Universidad Laica Eloy Alfaro de Manabí, Manta, Ecuador. 
El clima organizacional y su influencia en el desempeño académico de los docentes de la Universidad Laica Eloy Alfaro de Manabí

\title{
Resumen
}

El logro de una mayor productividad laboral docente, está en correspondencia al clima organizacional, el cual se gesta en las propias instituciones de educación superior, lo que hace necesario mantener de forma favorable dicha "correspondencia", sin embargo, es de notar en los docentes de la Universidad Laica Eloy Alfaro de Manabí (ULEAM), con absoluta frontalidad, el incumplimiento de algunos aspectos institucionales, tales como el escaso involucramiento laboral y comunicación interpersonal durante las funciones cotidianas. Con base a lo expuesto, esta investigación se planteó como objetivo determinar si el clima organizacional influye en el desempeño académico de los docentes de la ULEAM. Se utilizó el método inductivo y un diseño no experimental. La Población del estudio fue de 692 docentes con nombramiento, y la muestra de 247 catedráticos de acuerdo a la formula aplicada. La información se obtuvo a través de la aplicación de un instrumento tipo cuestionario en escala de Likert, y, el análisis de los datos se realizó bajo el uso del paquete estadístico SPSS. Los resultados indicaron de acuerdo al baremo de medición, que existe una correlación positiva y significativa entre el clima organizacional y el desempeño docente, significando que el "desempeño" de los profesores viene a estar afectado por el "clima organizacional" de la institución.

Palabras claves: Clima organizacional, desempeño laboral, docente con nombramientos y universidad.

\begin{abstract}
The achievement of greater teacher labor productivity, is in correspondence to the organizational climate, which is developed in the higher education institutions themselves, which makes it necessary to maintain a favorable "correspondence", however, it is noteworthy in the teachers of the Lay University Eloy Alfaro de Manabí (ULEAM), with absolute frontality, the breach of some institutional aspects, such as the scarce labor involvement and interpersonal communication during the daily functions. Based on the above, this research was aimed at determining if the organizational climate influences the academic performance of ULEAM teachers. The inductive method and a nonexperimental design were used. The study population was 692 teachers with appointment, and the sample of 247 professors according to the formula applied. The information was obtained through the
\end{abstract}


Edwin A. Delgado-Veliz; Marcos I. Granda-García; Temistocles D. Loor- Chávez; Wagner O. Villafuerte-Muñoz; Walter H. Villamarín Villota; Rafer Henry Cruz Mera

application of a Likert-scale questionnaire-type instrument, and the analysis of the data was made using the SPSS statistical package. The results indicated, according to the measurement scale, that there is a positive and significant correlation between the organizational climate and the teaching performance, meaning that the "performance" of the teachers is affected by the "organizational climate" of the institution.Keys words: Organizational climate, job performance, teacher with appointments and university.

\section{Resumo}

A conquista de uma maior produtividade do trabalho docente, está em correspondência com o clima organizacional, que se desenvolve nas próprias instituições de ensino superior, o que torna necessário manter uma "correspondência" favorável, no entanto, vale ressaltar nos professores da Universidade Leiga Eloy Alfaro de Manabí (ULEAM), com absoluta frontalidade, a quebra de alguns aspectos institucionais, como o escasso envolvimento laboral e a comunicação interpessoal durante as funções cotidianas. Com base no exposto, esta pesquisa teve como objetivo determinar se o clima organizacional influencia o desempenho acadêmico dos professores da ULEAM. O método indutivo e um desenho não experimental foram utilizados. A população do estudo foi de 692 professores com consulta e a amostra de 247 professores de acordo com a fórmula aplicada. As informações foram obtidas por meio da aplicação de um instrumento do tipo questionário da escala Likert, e a análise dos dados foi feita por meio do pacote estatístico SPSS. Os resultados indicaram, de acordo com a escala de medida, que existe uma correlação positiva e significativa entre o clima organizacional e o desempenho docente, significando que o "desempenho" dos professores é afetado pelo "clima organizacional" da instituição.

Palavras chave: Clima organizacional, desempenho no trabalho, ensino com consultas e universidade.

\section{Introducción.}

El clima organizacional, denominado también clima laboral, ambiente organizacional y/o laboral, cumple con la misión de lograr una mayor productividad y sobre todo mejorar los servicio 
El clima organizacional y su influencia en el desempeño académico de los docentes de la Universidad Laica Eloy Alfaro de Manabí

de la organizacion. El clima organizacional nos ayuda a detectar los problemas y resolverlos de

forma inmediatamente.

Según (Brunet, 1987), “el concepto de clima organizacional fue introducido por primera vez en la psicología industrial por Gellerman en el año de 1960”. Es por ello que influyo en el concepto de dos escuela de pensamiento tanto como la Gestalt y la escuela funcionalista. En la escuela de Gestalt comprende a los individuos basado en los criterios percibidos. Así como también los comportamientos de los individuos. Por otro lado la escuela funcionalista se basa en los pensamientos y comportamientos que rodea a los individuos dentro del ambiente que lo rodea.

Por lo cual el clima organizacional forma parte importante para cada uno de los trabajadores, ya que se pueden realizar diversos procesos tal y como los afirma (Duran, Atlante, \& Estela, 2010), “el clima organizacional es un proceso dinámico porque esta en constante estructuración y reestructuración".

A nivel mundial el clima organizacional está en constante cambio debidos a las políticas que rigen en cada uno de los países, ya que se contempla un plano de sometimiento jerárquico dentro de una organización.

Según (Barriga \& Rhomao, 2016) menciona que, "el clima organizacional en las universidades a nivel mundial, actualmente se encuentra sometidas a grandes retos y cambios constantes, producto del comportamiento de los escenarios donde actúan, debido a las innovaciones producidas por la globalización”.

Por otro lado (Duran, Atlante, \& Estela, 2010), menciona que países de latinoamericano tales como Venezuela, el estudio del clima organizacional surgió a partir de la década de los 80, por el interés de conocer los factores organizaciones. Se destaca el estudio realizado por (Perez y Sanabia, 
Edwin A. Delgado-Veliz; Marcos I. Granda-García; Temistocles D. Loor- Chávez; Wagner O. Villafuerte-Muñoz; Walter H. Villamarín Villota; Rafer Henry Cruz Mera

1997).

Para (Paco \& Matas, 2015), describe el clima organizacional de mucha relevancia para los docentes de instituciones públicas y privadas ya que en esta se puede observar conflictos laborales de diversa índole, permitiendo así el clima laboral desfavorable en el trabajo que se imparte.

Por otro lado (Calcina, 2012), menciona que es un asunto de importancia para aquellas organizaciones competitivas que buscan lograr una mayor productividad y mejora en el servicio ofrecido por medio de estrategias internas.

\section{Metodología.}

Para el presente trabajo se utilizó el método inductivo, debido a que se basa investigaciones micro para luego aplicarlas en lo macro.

De acuerdo a (Toro \& Parra, 2006) la investigación fue desarrollada con diseño no experimental debido a que se basa en observar fenómenos tal y como se dan naturalmente para después analizarlos.

La unidad de análisis de esta investigación es relacional debido a que se determinará si existe relación entre el clima organizacional y el desempeño académico de los docentes de la ULEAM.

\section{Resultados.}

La población de estudio corresponde al número de docentes de la Universidad Laica Eloy Alfaro de Manabí que tienen nombramiento y de acuerdo a estadísticas de la consulta de régimen laboral muestra una población de 692 docentes con nombramiento, obtenido de la página del departamento de talento humano de la ULEAM.

Se utilizaron encuestas en escala Likert, para determinar la relación entre las variables de la investigación. Para el efecto y fiabilidad de los resultados obtenidos se realizó el análisis de los datos en el programa SPSS, tomando un nivel de confianza del 95\% y un margen de error del 5\%, 
El clima organizacional y su influencia en el desempeño académico de los docentes de la Universidad Laica Eloy Alfaro de Manabí

con la finalidad de obtener mayor informacion en la evaluacion de los datos recogidos.

Para este caso se utilizó el muestreo probabilístico para determinar el tamaño de la misma.

$$
\begin{gathered}
n=\frac{\mathrm{Z}^{2} \mathrm{P} \mathrm{Q} N}{\mathrm{E}^{2}(\mathrm{~N}-1)+\mathrm{Z}^{2} \mathrm{P} \mathrm{Q}} \\
\mathrm{P}=50 \% \text { (probabilidad de que ocurra el evento) } \\
\mathrm{Q}=50 \% \text { (probabilidad de que no ocurra el evento) } \\
\mathrm{Z}^{2}=1.96 \text { (nivel de confianza) } \\
\mathrm{N}=\text { población (692) } \\
\mathrm{E}^{2}=0.05 \text { (Margen de error) } \\
\mathrm{n}=i ?(\text { tamaño de la muestra) } \\
n=n=\frac{(1.96)^{2} * 0.5 * 0.5 * 692}{0.05^{2}(692-1)+(1.96)^{2} * 0.5 * 0.5} \\
n=\frac{664.5968}{0.0025(691)+0,9604} \\
n=\frac{664.5968}{1.7275+0,9604} \\
n=\frac{664.5968}{2.6879} \\
n=247.25 \\
n=247 /
\end{gathered}
$$

\section{Estadístico de Cronbach.}

\begin{tabular}{|l|l|c|c|}
\hline \multicolumn{3}{|c|}{ Resumen de procesamiento de casos } \\
\hline \multirow{2}{*}{ Casos } & Válido & N & $\%$ \\
\cline { 2 - 4 } & Excluido & 247 & 100,0 \\
\cline { 2 - 4 } & Total & 247 &, 0 \\
\hline & & & 100,0 \\
\hline
\end{tabular}

Fuente: IBM SPSS Statistics 24

Elaborado por: Autores 
Edwin A. Delgado-Veliz; Marcos I. Granda-García; Temistocles D. Loor- Chávez; Wagner O. Villafuerte-Muñoz; Walter H. Villamarín Villota; Rafer Henry Cruz Mera

\begin{tabular}{|r|r|}
\hline \multicolumn{2}{|c|}{ Estadística de fiabilidad } \\
\hline $\begin{array}{c}\text { Alfa de } \\
\text { Cronbach }\end{array}$ & $\begin{array}{c}\text { N de } \\
\text { elementos }\end{array}$ \\
\hline, 576 & 35 \\
\hline
\end{tabular}

Fuente: IBM SPSS Statistics 24

Elaborado por: Autores

En el punto presente se constata que los datos retomados son confiables alcanzando una apreciación elevada en la escala de valoración del Alfa de Cronbach.

\begin{tabular}{|c|c|c|c|c|}
\hline \multicolumn{5}{|c|}{ Correlaciones Hipótesis General } \\
\hline & & & $\mathrm{X}$ & $\mathrm{Y}$ \\
\hline \multirow[t]{6}{*}{ Tau_b de Kendall } & \multirow{3}{*}{$\begin{array}{l}\text { CLIMA } \\
\text { ORGANIZACIONAL }\end{array}$} & Coeficiente de correlación & 1,000 &, $545^{* *}$ \\
\hline & & Sig. (bilateral) & &, 000 \\
\hline & & $\mathrm{N}$ & 247 & 247 \\
\hline & \multirow{3}{*}{$\begin{array}{l}\text { DESEMPEÑO } \\
\text { ACADÉMICO }\end{array}$} & Coeficiente de correlación &, $545^{* *}$ & 1,000 \\
\hline & & Sig. (bilateral) &, 000 & \\
\hline & & $\mathrm{N}$ & 247 & 247 \\
\hline \multirow[t]{6}{*}{ Rho de Spearman } & \multirow{3}{*}{$\begin{array}{l}\text { CLIMA } \\
\text { ORGANIZACIONAL }\end{array}$} & Coeficiente de correlación & 1,000 & $685^{* *}$ \\
\hline & & Sig. (bilateral) & & 000 \\
\hline & & $\mathrm{N}$ & 247 & 247 \\
\hline & \multirow{3}{*}{$\begin{array}{l}\text { DESEMPEÑO } \\
\text { ACADÉMICO }\end{array}$} & Coeficiente de correlación &, $685^{* *}$ & 1,000 \\
\hline & & Sig. (bilateral) &, 000 & \\
\hline & & $\mathrm{N}$ & 247 & 247 \\
\hline
\end{tabular}

Fuente: IBM SPSS Statistics 24

Elaborado por: Autores

Contraste de hipótesis: Se determinó la correlación conjunta del variable independiente $(\mathrm{x})$ clima organizacional y la variable dependiente (y) desempeño académico de los docentes de la Universidad Laica Eloy Alfaro de Manabí. Se halló una correlación moderada conjunta de 0,545**; y un Valor $\mathrm{p}=0,000$

Interpretación: Como el valor $\mathrm{p}=0.000<0.05$, se acepta que el clima organizacional influye el desempeño académico de los docentes de la Universidad Laica Eloy Alfaro de Manabí. Se puede confirmar que el estudio correlacional entre el "clima organizacional" y la variable dependiente: "desempeño académico", tienen una correlación positiva y significativa en el desempeño de los docentes de la universidad, quedando demostrado que se rechaza la hipótesis nula. 
El clima organizacional y su influencia en el desempeño académico de los docentes de la Universidad Laica Eloy Alfaro de Manabí

\begin{tabular}{|c|c|c|c|c|}
\hline \multicolumn{5}{|c|}{ Correlación Hipótesis Específica X1; Y1 } \\
\hline & & & $\mathrm{X} 1$ & Y1 \\
\hline \multirow[t]{6}{*}{ Tau_b de Kendall } & \multirow{3}{*}{$\begin{array}{l}\text { ASPECTO } \\
\text { INSTITUCIONAL }\end{array}$} & Coeficiente de correlación & 1,000 &, $595^{* *}$ \\
\hline & & Sig. (bilateral) &. & ,000 \\
\hline & & $\mathrm{N}$ & 247 & 247 \\
\hline & \multirow{3}{*}{$\begin{array}{l}\text { FUNCIÓN } \\
\text { PROFESIONAL }\end{array}$} & Coeficiente de correlación &, $595^{* *}$ & 1,000 \\
\hline & & Sig. (bilateral) & ,000 & ${ }^{\circ}$ \\
\hline & & $\mathrm{N}$ & 247 & 247 \\
\hline \multirow[t]{6}{*}{ Rho de Spearman } & \multirow{3}{*}{$\begin{array}{l}\text { ASPECTO } \\
\text { INSTITUCIONAL }\end{array}$} & Coeficiente de correlación & 1,000 & $679^{* *}$ \\
\hline & & Sig. (bilateral) & . & ,000 \\
\hline & & $\mathrm{N}$ & 247 & 247 \\
\hline & \multirow{3}{*}{$\begin{array}{l}\text { FUNCIÓN } \\
\text { PROFESIONAL }\end{array}$} & Coeficiente de correlación &, $679^{* *}$ & 1,000 \\
\hline & & Sig. (bilateral) &, 000 & 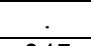 \\
\hline & & $\mathrm{N}$ & 247 & 247 \\
\hline
\end{tabular}

Fuente: IBM SPSS Statistics 24

Elaborado por: Autores

Contraste de hipótesis: Se determinó la correlación conjunta del variable independiente $(\mathrm{x})$ clima organizacional con la dimensión aspecto institucional y la dimensión función profesional de los docentes de la Universidad Laica Eloy Alfaro de Manabí. Se halló una correlación conjunta moderada de 0, 595**; y un Valor $\mathrm{p}=0,000$.

Interpretación: Como el valor $\mathrm{p}=0.000<0.05$, se acepta que el clima organizacional influye el desempeño académico de los docentes de la Universidad Laica Eloy Alfaro de Manabí. Se puede confirmar que el estudio correlacional entre el "aspecto institucional" y la variable dependiente: "función profesional", tienen una correlación positiva y significativa en el desempeño de los docentes de la universidad, quedando demostrado que se rechaza la hipótesis nula.

\begin{tabular}{|c|c|c|c|c|}
\hline \multicolumn{5}{|c|}{ Correlación Hipótesis Específica X1; Y2 } \\
\hline & & & $\mathrm{X} 1$ & $\mathrm{Y} 2$ \\
\hline \multirow[t]{6}{*}{ Tau_b de Kendall } & \multirow{3}{*}{$\begin{array}{l}\text { ASPECTO } \\
\text { INSTITUCIONAL }\end{array}$} & Coeficiente de correlación & 1,000 &, $503^{* *}$ \\
\hline & & Sig. (bilateral) & &, 000 \\
\hline & & $\mathrm{N}$ & 247 & 247 \\
\hline & \multirow{3}{*}{$\begin{array}{l}\text { SISTEMA DE } \\
\text { EVALUACIÓN }\end{array}$} & Coeficiente de correlación &, $503^{* *}$ & 1,000 \\
\hline & & Sig. (bilateral) & 000 & \\
\hline & & $\mathrm{N}$ & 247 & 247 \\
\hline \multirow[t]{6}{*}{ Rho de Spearman } & \multirow{3}{*}{$\begin{array}{l}\text { ASPECTO } \\
\text { INSTITUCIONAL }\end{array}$} & Coeficiente de correlación & 1,000 & $678^{* *}$ \\
\hline & & Sig. (bilateral) & 8 &, 000 \\
\hline & & $\mathrm{N}$ & 247 & 247 \\
\hline & \multirow{3}{*}{$\begin{array}{l}\text { SISTEMA DE } \\
\text { EVALUACIÓN }\end{array}$} & Coeficiente de correlación & $678^{* *}$ & 1,000 \\
\hline & & Sig. (bilateral) & 000 & \\
\hline & & $\mathrm{N}$ & 247 & 247 \\
\hline
\end{tabular}

Fuente: IBM SPSS Statistics 24

Elaborado por: Autores 
Edwin A. Delgado-Veliz; Marcos I. Granda-García; Temistocles D. Loor- Chávez; Wagner O. Villafuerte-Muñoz; Walter H. Villamarín Villota; Rafer Henry Cruz Mera

Contraste de hipótesis: Se determinó la correlación conjunta del variable independiente (x) clima organizacional con la dimensión aspecto institucional y la dimensión sistema de evaluación de los docentes de la Universidad Laica Eloy Alfaro de Manabí. Se halló una correlación conjunta moderada de $0,503^{* *}$; y un Valor $\mathrm{p}=0,000$.

Interpretación: Como el valor $\mathrm{p}=0.000<0.05$, se acepta que el clima organizacional influye el desempeño académico de los docentes de la Universidad Laica Eloy Alfaro de Manabí. Se puede confirmar que el estudio correlacional entre el "aspecto institucional" y la variable dependiente: "sistema de evaluación", tienen una correlación positiva y significativa en el desempeño de los docentes de la universidad, quedando demostrado que se rechaza la hipótesis nula.

\begin{tabular}{|c|c|c|c|c|}
\hline \multicolumn{5}{|c|}{ Correlación Hipótesis Específica X1; Y3 } \\
\hline & & & $\mathrm{X} 1$ & $\mathrm{Y3}$ \\
\hline \multirow[t]{6}{*}{ Tau_b de Kendall } & \multirow{3}{*}{$\begin{array}{l}\text { ASPECTO } \\
\text { INSTITUCIONAL }\end{array}$} & Coeficiente de correlación & 1,000 &, $544^{* *}$ \\
\hline & & Sig. (bilateral) & &, 000 \\
\hline & & $\mathrm{N}$ & 247 & 247 \\
\hline & \multirow{3}{*}{$\begin{array}{l}\text { CAPITAL } \\
\text { HUMANO }\end{array}$} & Coeficiente de correlación &, $544^{* *}$ & 1,000 \\
\hline & & Sig. (bilateral) &, 000 & \\
\hline & & $\mathrm{N}$ & 247 & 247 \\
\hline \multirow[t]{6}{*}{ Rho de Spearman } & \multirow{3}{*}{$\begin{array}{l}\text { ASPECTO } \\
\text { INSTITUCIONAL }\end{array}$} & Coeficiente de correlación & 1,000 & $597^{* *}$ \\
\hline & & Sig. (bilateral) & &, 000 \\
\hline & & $\mathrm{N}$ & 247 & 247 \\
\hline & \multirow{3}{*}{$\begin{array}{l}\text { CAPITAL } \\
\text { HUMANO }\end{array}$} & Coeficiente de correlación &, $597^{* *}$ & 1,000 \\
\hline & & Sig. (bilateral) & ,000 & \\
\hline & & $\mathrm{N}$ & 247 & 247 \\
\hline
\end{tabular}

Fuente: IBM SPSS Statistics 24

Elaborado por: Autores

Contraste de hipótesis: Se determinó la correlación conjunta del variable independiente $(\mathrm{x})$ clima organizacional con la dimensión aspecto institucional y la dimensión capital humano de los docentes de la Universidad Laica Eloy Alfaro de Manabí. Se halló una correlación conjunta moderada de $0,544 * *$; y un Valor $\mathrm{p}=0,000$.

Interpretación: Como el valor $\mathrm{p}=0.000<0.05$, se acepta que el clima organizacional influye el desempeño académico de los docentes de la Universidad Laica Eloy Alfaro de Manabí. Se puede confirmar que el estudio correlacional entre el "aspecto institucional" y la variable dependiente: "capital humano", tienen una correlación positiva y significativa en el desempeño de los docentes de la universidad, quedando demostrado que se rechaza la hipótesis nula. 
El clima organizacional y su influencia en el desempeño académico de los docentes de la Universidad Laica Eloy Alfaro de Manabí

\begin{tabular}{|c|c|c|c|c|}
\hline \multicolumn{5}{|c|}{ Correlación Hipótesis Específica X2; Y1 } \\
\hline & & & $\mathrm{X} 2$ & Y1 \\
\hline \multirow[t]{6}{*}{ Tau_b de Kendall } & \multirow{3}{*}{$\begin{array}{l}\text { INVOLUCRAMIENTO } \\
\text { LABORAL }\end{array}$} & Coeficiente de correlación & 1,000 &, $573^{* *}$ \\
\hline & & Sig. (bilateral) & . & 000 \\
\hline & & $\mathrm{N}$ & 247 & 247 \\
\hline & \multirow{3}{*}{$\begin{array}{l}\text { FUNCIÓN } \\
\text { PROFESIONAL }\end{array}$} & Coeficiente de correlación &, $573^{* *}$ & 1,000 \\
\hline & & Sig. (bilateral) &, 000 & \\
\hline & & $\mathrm{N}$ & 247 & 247 \\
\hline \multirow[t]{6}{*}{ Rho de Spearman } & \multirow{3}{*}{$\begin{array}{l}\text { INVOLUCRAMIENTO } \\
\text { LABORAL }\end{array}$} & Coeficiente de correlación & 1,000 & ,640** \\
\hline & & Sig. (bilateral) & & ,000 \\
\hline & & $\mathrm{N}$ & 247 & 247 \\
\hline & \multirow{3}{*}{$\begin{array}{l}\text { FUNCIÓN } \\
\text { PROFESIONAL }\end{array}$} & Coeficiente de correlación &, $640^{* *}$ & 1,000 \\
\hline & & Sig. (bilateral) & ,000 & \\
\hline & & $\mathrm{N}$ & 247 & 247 \\
\hline
\end{tabular}

Fuente: IBM SPSS Statistics 24

Elaborado por: Autores

Contraste de hipótesis: Se determinó la correlación conjunta del variable independiente $(\mathrm{x})$ clima organizacional con la dimensión involucramiento laboral y la dimensión función profesional de los docentes de la Universidad Laica Eloy Alfaro de Manabí. Se halló una correlación conjunta moderada de $0,573 * *$; y un Valor $\mathrm{p}=0,000$.

Interpretación: Como el valor $\mathrm{p}=0.000<0.05$, se acepta que el clima organizacional influye el desempeño académico de los docentes de la Universidad Laica Eloy Alfaro de Manabí. Se puede confirmar que el estudio correlacional entre el "involucramiento laboral" y la variable dependiente: "función profesional", tienen una correlación positiva y significativa en el desempeño de los docentes de la universidad, quedando demostrado que se rechaza la hipótesis nula.

\begin{tabular}{|c|c|c|c|c|}
\hline \multicolumn{5}{|c|}{ Correlación Hipótesis Específica X2; Y2 } \\
\hline & & & $\mathrm{X} 2$ & $\mathrm{Y} 2$ \\
\hline \multirow[t]{6}{*}{ Tau_b de Kendall } & \multirow{3}{*}{$\begin{array}{l}\text { INVOLUCRAMIENTO } \\
\text { LABORAL }\end{array}$} & Coeficiente de correlación & 1,000 &, $572^{* *}$ \\
\hline & & Sig. (bilateral) & & 000 \\
\hline & & $\mathrm{N}$ & 247 & 247 \\
\hline & \multirow{3}{*}{$\begin{array}{l}\text { SISTEMA DE } \\
\text { EVALUACIÓN }\end{array}$} & Coeficiente de correlación &, $572^{* *}$ & 1,000 \\
\hline & & Sig. (bilateral) & 000 & \\
\hline & & $\mathrm{N}$ & 247 & 247 \\
\hline \multirow[t]{6}{*}{ Rho de Spearman } & \multirow{3}{*}{$\begin{array}{l}\text { INVOLUCRAMIENTO } \\
\text { LABORAL }\end{array}$} & Coeficiente de correlación & 1,000 &, $629^{* *}$ \\
\hline & & Sig. (bilateral) & &, 000 \\
\hline & & $\mathrm{N}$ & 247 & 247 \\
\hline & \multirow{3}{*}{$\begin{array}{l}\text { SISTEMA DE } \\
\text { EVALUACIÓN }\end{array}$} & Coeficiente de correlación &, $629^{* *}$ & 1,000 \\
\hline & & Sig. (bilateral) &, 000 & \\
\hline & & $\mathrm{N}$ & 247 & 247 \\
\hline
\end{tabular}

Fuente: IBM SPSS Statistics 24

Elaborado por: Autores

Pol. Con. (Edición núm. 28) Vol. 3, No 12, diciembre 2018, pp. 428-445, ISSN: 2550 - 682X 
Edwin A. Delgado-Veliz; Marcos I. Granda-García; Temistocles D. Loor- Chávez; Wagner O. Villafuerte-Muñoz; Walter H. Villamarín Villota; Rafer Henry Cruz Mera

Contraste de hipótesis: Se determinó la correlación conjunta del variable independiente (x) clima organizacional con la dimensión involucramiento laboral y la dimensión sistema de evaluación de los docentes de la Universidad Laica Eloy Alfaro de Manabí. Se halló una correlación conjunta moderada de $0,572 *$; y un Valor $\mathrm{p}=0,000$.

Interpretación: Como el valor $\mathrm{p}=0.000<0.05$, se acepta que el clima organizacional influye el desempeño académico de los docentes de la Universidad Laica Eloy Alfaro de Manabí. Se puede confirmar que el estudio correlacional entre el "involucramiento laboral" y la variable dependiente: "sistema de evaluación", tienen una correlación positiva y significativa en el desempeño de los docentes de la universidad, quedando demostrado que se rechaza la hipótesis nula.

\begin{tabular}{|c|c|c|c|c|}
\hline \multicolumn{5}{|c|}{ Correlación Hipótesis Específica X2; Y3 } \\
\hline & & & $\mathrm{X} 2$ & $\mathrm{Y} 3$ \\
\hline \multirow[t]{6}{*}{ Tau_b de Kendall } & \multirow{3}{*}{$\begin{array}{l}\text { INVOLUCRAMIENTO } \\
\text { LABORAL }\end{array}$} & Coeficiente de correlación & 1,000 &, $553^{* *}$ \\
\hline & & Sig. (bilateral) & &, 000 \\
\hline & & $\mathrm{N}$ & 247 & 247 \\
\hline & \multirow{3}{*}{ CAPITAL HUMANO } & Coeficiente de correlación &, $553^{* *}$ & 1,000 \\
\hline & & Sig. (bilateral) &, 000 & \\
\hline & & $\mathrm{N}$ & 247 & 247 \\
\hline \multirow{6}{*}{ Rho de Spearman } & \multirow{3}{*}{$\begin{array}{l}\text { INVOLUCRAMIENTO } \\
\text { LABORAL }\end{array}$} & Coeficiente de correlación & 1,000 &, $600^{* *}$ \\
\hline & & Sig. (bilateral) & &, 000 \\
\hline & & $\mathrm{N}$ & 247 & 247 \\
\hline & \multirow[t]{3}{*}{ CAPITAL HUMANO } & Coeficiente de correlación &, $600^{* *}$ & 1,000 \\
\hline & & Sig. (bilateral) & 000 & \\
\hline & & $\mathrm{N}$ & 247 & 247 \\
\hline
\end{tabular}

Fuente: IBM SPSS Statistics 24

Elaborado por: Autores

Contraste de hipótesis: Se determinó la correlación conjunta del variable independiente $(\mathrm{x})$ clima organizacional con la dimensión involucramiento laboral y la dimensión capital humano de los docentes de la Universidad Laica Eloy Alfaro de Manabí. Se halló una correlación conjunta moderada de $0,553^{* *}$; y un Valor $\mathrm{p}=0,000$.

Interpretación: Como el valor $\mathrm{p}=0.000<0.05$, se acepta que el clima organizacional influye el desempeño académico de los docentes de la Universidad Laica Eloy Alfaro de Manabí. Se puede confirmar que el estudio correlacional entre el "involucramiento laboral" y la variable dependiente: "capital humano", tienen una correlación positiva y significativa en el desempeño de los docentes de la universidad, quedando demostrado que se rechaza la hipótesis nula. 
El clima organizacional y su influencia en el desempeño académico de los docentes de la Universidad Laica Eloy Alfaro de Manabí

\begin{tabular}{|c|c|c|c|c|}
\hline \multicolumn{5}{|c|}{ Correlacion Hipotesis Especifica X3; YI } \\
\hline & & & $\mathrm{X} 3$ & Y1 \\
\hline \multirow{6}{*}{ Tau_b de Kendall } & \multirow{3}{*}{$\begin{array}{l}\text { COMUNICACIÓN } \\
\text { INTERPERSONAL }\end{array}$} & Coeficiente de correlación & 1,000 &, $548^{* *}$ \\
\hline & & Sig. (bilateral) & &, 000 \\
\hline & & $\mathrm{N}$ & 247 & 247 \\
\hline & \multirow{3}{*}{$\begin{array}{l}\text { FUNCIÓN } \\
\text { PROFESIONAL }\end{array}$} & Coeficiente de correlación &, $548^{* *}$ & 1,000 \\
\hline & & Sig. (bilateral) &, 000 & \\
\hline & & $\mathrm{N}$ & 247 & 247 \\
\hline \multirow{6}{*}{ Rho de Spearman } & \multirow{3}{*}{$\begin{array}{l}\text { COMUNICACIÓN } \\
\text { INTERPERSONAL }\end{array}$} & Coeficiente de correlación & 1,000 &, $646^{* *}$ \\
\hline & & Sig. (bilateral) & &, 000 \\
\hline & & $\mathrm{N}$ & 247 & 247 \\
\hline & \multirow{3}{*}{$\begin{array}{l}\text { FUNCIÓN } \\
\text { PROFESIONAL }\end{array}$} & Coeficiente de correlación &, $646^{* *}$ & 1,000 \\
\hline & & Sig. (bilateral) &, 000 & \\
\hline & & $\mathrm{N}$ & 247 & 247 \\
\hline
\end{tabular}

Fuente: IBM SPSS Statistics 24

Elaborado por: Autores

Contraste de hipótesis: Se determinó la correlación conjunta del variable independiente (x) clima organizacional con la dimensión comunicación interpersonal y la dimensión función profesional de los docentes de la Universidad Laica Eloy Alfaro de Manabí. Se halló una correlación conjunta moderada de $0,548^{* *}$; y un Valor $\mathrm{p}=0,000$.

Interpretación: Como el valor $\mathrm{p}=0.000<0.05$, se acepta que el clima organizacional influye el desempeño académico de los docentes de la Universidad Laica Eloy Alfaro de Manabí. Se puede confirmar que el estudio correlacional entre el "comunicación interpersonal" y la variable dependiente: "función profesional", tienen una correlación positiva y significativa en el desempeño de los docentes de la universidad, quedando demostrado que se rechaza la hipótesis nula.

\begin{tabular}{|c|c|c|c|c|}
\hline \multicolumn{5}{|c|}{ Correlación Hipótesis Específica X3; Y2 } \\
\hline & & & $\mathrm{X} 3$ & Y2 \\
\hline \multirow[t]{6}{*}{ Tau_b de Kendall } & \multirow{3}{*}{$\begin{array}{l}\text { COMUNICACIÓN } \\
\text { INTERPERSONAL }\end{array}$} & Coeficiente de correlación & 1,000 &, $542^{* *}$ \\
\hline & & Sig. (bilateral) & &, 000 \\
\hline & & $\mathrm{N}$ & 247 & 247 \\
\hline & \multirow{3}{*}{$\begin{array}{l}\text { SISTEMA DE } \\
\text { EVALUACIÓN }\end{array}$} & Coeficiente de correlación &, $542^{* *}$ & 1,000 \\
\hline & & Sig. (bilateral) & 000 & \\
\hline & & $\mathrm{N}$ & 247 & 247 \\
\hline \multirow[t]{6}{*}{ Rho de Spearman } & \multirow{3}{*}{$\begin{array}{l}\text { COMUNICACIÓN } \\
\text { INTERPERSONAL }\end{array}$} & Coeficiente de correlación & 1,000 &, $646^{* *}$ \\
\hline & & Sig. (bilateral) & &, 000 \\
\hline & & $\mathrm{N}$ & 247 & 247 \\
\hline & \multirow{3}{*}{$\begin{array}{l}\text { SISTEMA DE } \\
\text { EVALUACIÓN }\end{array}$} & Coeficiente de correlación &, $646^{* *}$ & 1,000 \\
\hline & & Sig. (bilateral) &, 000 & \\
\hline & & $\mathrm{N}$ & 247 & 247 \\
\hline
\end{tabular}

Fuente: IBM SPSS Statistics 24

Elaborado por: Autores 
Edwin A. Delgado-Veliz; Marcos I. Granda-García; Temistocles D. Loor- Chávez; Wagner O. Villafuerte-Muñoz; Walter H. Villamarín Villota; Rafer Henry Cruz Mera

Contraste de hipótesis: Se determinó la correlación conjunta del variable independiente (x) clima organizacional con la dimensión comunicación interpersonal y la dimensión sistema de evaluación de los docentes de la Universidad Laica Eloy Alfaro de Manabí. Se halló una correlación conjunta moderada de $0,542 * *$; yn Valor $\mathrm{p}=0,000$.

Interpretación: Como el valor $\mathrm{p}=0.000<0.05$, se acepta que el clima organizacional influye el desempeño académico de los docentes de la Universidad Laica Eloy Alfaro de Manabí. Se puede confirmar que el estudio correlacional entre el "comunicación interpersonal" y la variable dependiente: "sistema de evaluación", tienen una correlación positiva y significativa en el desempeño de los docentes de la universidad, quedando demostrado que se rechaza la hipótesis nula.

\begin{tabular}{|c|c|c|c|c|}
\hline \multicolumn{5}{|c|}{ Correlación Hipótesis Específica X3; Y3 } \\
\hline & & & $\mathrm{X} 3$ & $\mathrm{Y3}$ \\
\hline \multirow[t]{6}{*}{ Tau_b de Kendall } & \multirow{3}{*}{$\begin{array}{l}\text { COMUNICACIÓN } \\
\text { INTERPERSONAL }\end{array}$} & Coeficiente de correlación & 1,000 &, $593^{* *}$ \\
\hline & & Sig. (bilateral) & &, 000 \\
\hline & & $\mathrm{N}$ & 247 & 247 \\
\hline & \multirow{3}{*}{$\begin{array}{l}\text { CAPITAL } \\
\text { HUMANO }\end{array}$} & Coeficiente de correlación &, $593^{* *}$ & 1,000 \\
\hline & & Sig. (bilateral) &, 000 & \\
\hline & & $\mathrm{N}$ & 247 & 247 \\
\hline \multirow[t]{6}{*}{ Rho de Spearman } & \multirow{3}{*}{$\begin{array}{l}\text { COMUNICACIÓN } \\
\text { INTERPERSONAL }\end{array}$} & Coeficiente de correlación & 1,000 & ,672 \\
\hline & & Sig. (bilateral) & &, 000 \\
\hline & & $\mathrm{N}$ & 247 & 247 \\
\hline & \multirow{3}{*}{$\begin{array}{l}\text { CAPITAL } \\
\text { HUMANO }\end{array}$} & Coeficiente de correlación & ,672 & 1,000 \\
\hline & & Sig. (bilateral) &, 000 & \\
\hline & & $\mathrm{N}$ & 247 & 247 \\
\hline
\end{tabular}

Fuente:IBM SPSS Statistics 24

Elaborado por: Autores

Contraste de hipótesis: Se determinó la correlación conjunta del variable independiente $(\mathrm{x})$ clima organizacional con la dimensión comunicación interpersonal y la dimensión capital humano de los docentes de la Universidad Laica Eloy Alfaro de Manabí. Se halló una correlación conjunta moderada de $0,593^{* *}$; $\mathrm{y}$ un Valor $\mathrm{p}=0,000$.

Interpretación: Como el valor $\mathrm{p}=0.000<0.05$, se acepta que el clima organizacional influye el desempeño académico de los docentes de la Universidad Laica Eloy Alfaro de Manabí. Se puede confirmar que el estudio correlacional entre el "comunicación interpersonal" y la variable dependiente: "capital humano", tienen una correlación positiva y significativa en el desempeño de los docentes de la universidad, quedando demostrado que se rechaza la hipótesis nula. 
El clima organizacional y su influencia en el desempeño académico de los docentes de la Universidad Laica Eloy Alfaro de Manabí

\section{Conclusiones.}

1. Se determina que el clima organizacional si influye en el desenvolvimiento de los docentes dentro de la institución de la universidad.

2. La hipótesis que se plantearon determinaron que los análisis de correlación y la confrontación de las hipótesis en el desempeño académico es moderado, las cuales tiene una correlación positiva en cuanto la participación del docentes dentro de la universidad.

3. Se determinó que las instalaciones en donde se involucra el docente influye mucho para el mejor desenvolvimiento tanto académico y/o pedagógico.

4. Dentro de la variable desempeño académico en la recolección y análisis, se puede obtener que se debe incluir más en la participación dentro del personal administrativo hacia los docentes.

5. Los docentes mencionan que cada uno de ellos se desempeña arduamente para conseguir un alto rendimiento en la institución, es por ello que trabajan en conjunto para conseguir el éxito esperado por las autoridades.

6. Los docentes encuestados tiene un porcentaje de $38,9 \%$ en participación de actividades académicos en otra institución nacional, la cuales permite el alto involucramiento académico en la investigación científica y sus resultados obtenidos de su investigación previamente realizada. 
Edwin A. Delgado-Veliz; Marcos I. Granda-García; Temistocles D. Loor- Chávez; Wagner O. Villafuerte-Muñoz; Walter H. Villamarín Villota; Rafer Henry Cruz Mera

\section{Bibliografía.}

Alonzo, C., Estévez, M., Guarinoni, C., Day, A., \& Paula, Q. (2008). Construciòn de un cuestionario de clima organizacional. Dimensiones de anàlisis. XV Jornadas de Investigación y Cuarto Encuentro de Investigadores en Psicología.

Barriga, R., \& Rhomao, D. (2016). Clima organizacional y desempeño docente en la Universidad “Jaime Bausate y Meza” Jesús María - Lima 2016. Lima - Perù.

Barrón, M. (2009). Docencia universitaria y competencias didácticas. Perfiles Educativos , 76-87.

Blanch, F., \& Luis, M. (2002). Aspectos institucionales del desarrollo sostenible. Resvista ICE, 7-14.

Brunet, L. (1987). El clima de trabajo en las organizaciones: Definición, diagnóstico y consecuencias. Mexico : Trillas.

Calcina, Y. (2012). El clima institucional y su incidencia en el desempeño laboral de los docentes de la facultad de ciencias sociales de la Universidad Nacional del Altiplano y facultad de ciencias de la educaciòn Universidad Andina. Revista de Investigación en Comunicación y Desarrollo, p. 23.

Chiavenato, I. (2007). Administración de recursos humanos - El capital humano de las organizaciones. Mexico : McGRAW-HILL.

Duran, C., Atlante, M., \& Estela, G. (2010). El clima organizacional en al universidad: Modelo de medicion para el personal de apoyo universitario.

García, E. (2011). Clima organizacional basado en la gestión del conocimiento en el sector universitario público autónomo. Venezuela.

González, H. (2013). Evaluación del desempeño docente: experiencia en una universidad ecuatoriana. Quito-Eciuador: Senefelder.

ICFES. (2014). Determinantes del desempeño. Colombia: ICFES.

LOES. (2016). Ley Organica de Educaciòn Superior. Quito-Ecuador.

Loor, T. D. (2018). El clima organizacional y su incidencia en la formación profesional del docente en las instituciones de educación superior (IES) de la provincia de Manabí, República del Ecuador. Lima-Perù.

Madrigal, B. (2009). Capital humano e intelectual: su evaluaciòn. Venezuela.

Mejías, A., Reyes, O., \& Arzola, M. (2006). Medición del clima organizacional en instituciones de educación superior. Universidad, Ciencia y Tecnología. 
El clima organizacional y su influencia en el desempeño académico de los docentes de la Universidad Laica Eloy Alfaro de Manabí

Moreira, L. (2016). Clima organizacional en la Eduacion Superior, Ecuador. Dominıo de las clenclas , 296-307.

Paco, R., \& Matas, J. (2015). Clima organizacional y desempeño laboral docente en instituciones educativas de nivel primario y secundario. Apuntes de Ciencia \& Sociedad, p.335.

Quintero, M., \& German, O. (2013). El desempeño académico: una opción para la cualificación de las instituciones educativas. Plumilla Educativa , pp. 93-115.

Quispe, E. (2015). Clima organizacional y desempeño laboral en la municipalidad distrital de pacucha, andahuaylas, 2015. Andahuaylas, Perú.

Rosario, D. (2016). Clima organizacional y nivel de compromiso laboral de los trabajadores de la Empresa de Servicios Postales del Perú. Lima 2016. Lima.

Segredo, A. (2003). Percepción del Clima Organizacional por Directores de policlínicos. Escuela Nacional de Salud Pública de Cuba., Cuba.

Solar, M., \& Josè, S. (2007). Desempeño docente desde la perspectiva del enfoque profesional. Chile.

Toro, I., \& Parra, R. (2006). Método y Conocimiento: Metodología de la Imvestigación (5 ed.). Medellín: EAFIT. 